\title{
Botulinum toxin injection for the treatment of epiphora in lacrimal outflow obstruction
}

\begin{abstract}
Purpose: To describe our experience with the use of botulinum toxin (BoNTA) for the symptomatic treatment of lacrimal outflow obstruction.

Methods: We retrospectively reviewed the case notes of patients with epiphora due to lacrimal outflow obstruction who chose to have injections of botulinum toxin into the palpebral lobe of the lacrimal gland instead of surgery between 2009 and 2014. Epiphora was graded subjectively with Munk scores obtained before and after treatment as well as qualitative degree of improvement reported by the patients. Severity and duration of side effects were also noted.
\end{abstract}

Results: Seventeen patients (22 eyes, mean age $70.3,4$ males and 13 females) were identified. A mean of 3.5 (range; 1-10) injections of BoNTA (Botox, Allergan; 1.25-7.5 units) were given per eye. The mean interval between injections was 3.9 months (range 3-6). The mean Munk score (3.4, range 2-4) improved significantly after treatment to 1.6 (range: 0-3, $P=0.0001$ paired two-tailed $t$-test). Epiphora completely resolved in a fifth, improved by up to $60-90 \%$ in a half and only 'a little better' in a further fifth. Temporary bruising and diplopia (lasting 2 weeks) was reported in $12 \%(2 / 17)$.

Conclusion: We report our outcomes for BoNTA to the palpebral lobe of the lacrimal gland in patients with lacrimal outflow obstruction epiphora seeking alternatives to surgery. This data provide further evidence for informed consent and for commissioning organisations considering the funding of this treatment. Eye (2015) 29, 656-661; doi:10.1038/eye.2015.18; published online 6 March 2015

\section{Introduction}

Accepted in revised form: 8 January 2015

Published online:

6 March 2015
Epiphora owing to lacrimal outflow obstruction affects a wide range of patients. It adversely
K Ziahosseini, Z Al-Abbadi and R Malhotra

affects their quality of life by causing blurred vision, irritation of the eye and periocular tissue and social embarrassment. Its standard treatment is surgical in the form of DCR (dacryocystorhinstomy) and even Jones tube intubation depending upon the location and extent of obstruction. Jones tubes, however, have a high rate of displacement and the need for further procedures and patient satisfaction does not always correlate with the anatomical success. ${ }^{1-3}$ Surgery may not be the best option in all patients especially in the elderly population. It may also be medically inadvisable in those who have undergone excision of lacrimal apparatus for treatment of malignant lesions affecting the neighbouring anatomical structures.

The main and accessory lacrimal glands both significantly contribute to basal and reflex tear production. ${ }^{4}$ Injection of botulinum toxin A (BoNTA) in the lacrimal gland blocks the presynaptic release of acetylcholine, which is required for tear secretion.

BoNTA has been successfully used to treat excess tear production in the setting of gustatory hyperlacrimation owing to aberrant regeneration in proximal facial nerve injuries. ${ }^{5-9}$ To our knowledge, only two publications report its use in the treatment of either functional epiphora (14 patients patent to syringing) ${ }^{10}$ or lacrimal obstruction (27 patients). ${ }^{9}$

We report the outcomes of BoNTA injections in a group of patients with lacrimal outflow obstruction owing to a variety of pathologies.

\section{Methods}

We retrospectively reviewed the case notes of 17 patients with epiphora owing to lacrimal outflow obstruction who chose to have injections of BoNTA into the palpebral lobe of the lacrimal gland instead of surgery at a single centre 
between 2009-2014. IRB approval for this audit was obtained. Epiphora was graded subjectively with Munk scores before and after treatment. ${ }^{11}$ Patients were also asked to report any improvement on a scale of zero to hundred per cent and to report if their symptoms were 'worse', ' no better', 'a little better', 'a lot better but not completely' or 'completely better' after the injection. The severity and duration of side effects were also noted.

BoNTA (Botox, Allergan) was reconstituted with sterile, preservative-free $0.9 \%$ sodium chloride solution diluted to a concentration of 50 units $/ 1 \mathrm{ml}$. Each patient received topical Oxybuprocaine Hydrochloride 0.4\% w/v (Bausch \& Lomb, Kingston-upon-Thames, UK) before injection. The lateral upper eyelid was manually distracted away from the globe, while the patient looked down and to the opposite side to expose the palpebral lobe of the lacrimal gland. A transconjunctival injection of 1.25-7.5 units of BoNTA (the most common dose being 2.5 units) using a 27-gauge needle on a $1-\mathrm{ml}$ syringe was given into the palpebral lobe.

\section{Results}

A total of 22 eyes of 17 patients were identified, 4 males, 13 females. The mean age was 70.3 (median $=72$, range 36-93). Twelve eyes (10 patients) had canalicular or common canalicular obstruction, four eyes (3 patients) had nasolacrimal duct (NLD) obstruction and six eyes (4 patients) developed epiphora after punctal cautery. (Table 1) Sixteen patients had troublesome epiphora; one patient had bilateral blurred vision owing to a high tear meniscus encroaching upon the visual axis.

A total of 73 injections were given, 19 by a consultant and 54 by fellows. A mean of 3.5 injections (median $=3$, range 1-10) were given per eye. Seven eyes (5 patients) had $>3$ injections.

The median duration of effectiveness was 10 weeks (range 3-24 weeks). The mean indoors Munk score (3.4, range $2-4$ ) improved significantly after treatment to 1.6 (range $0-4, P=0.00001$, paired two-tailed $t$-test).

The mean outdoors Munk score (3.8, range 3-4) improved significantly after treatment to 1.7 (range $0-3, P=0.0001$; Table 2).

Three patients $(18 \%, 3$ eyes) reported their epiphora completely resolved, 53\% (9/17, 12 eyes) patients reported their epiphora was a lot better but not completely resolved (60-90\% improvement). Three (18\%, 5 eyes) were 'a little better' (30-50\% improvement), one patient (1 eye) did not report any subjective improvement after two treatments and one patient (1 eye) deteriorated after one injection (further described below). Five patients received bilateral treatment of similar dose and they reported similar outcomes of the two sides (Table 3).
The mean interval between injections was 3.9 months (range 3-6). One patient developed a lacrimal gland and upper eyelid haematoma following injection that lasted 2 weeks. Another patient had horizontal diplopia that prevented her from driving for 2 weeks. (Table 2)

Sixteen eyes (13 patients) received 2.5 units at every treatment. Four eyes (3 patients) received 5-7.5 units. One patient had 1.25 units bilaterally at her first treatment that was later increased to 2.5 units. Early on, during our learning curve, three patients received 5-7.5 units. One patient with canalicular stenosis received 7.5 units at her first treatment with $80 \%$ improvement. She was given 5 and then 2.5 units in her subsequent treatments with similar degree of effectiveness and duration. Two other patients also received 5 units with $60-90 \%$ improvement. None experienced any side effects. Therefore, we recommend a starting dose of 2.5 units.

One patient had four-lid punctal cautery for dry eyes syndrome (reduced Schirmer's with anaesthetic of 2-3 $\mathrm{mm}$ after $5 \mathrm{~min}$ ) and despite improvement in the ocular surface with a reduction in corneal punctate staining and no objective evidence of tear overflow, had symptomatic epiphora. She underwent three BoNTA $(1.25,2.5$ and 2.5 units) treatments over the course of a year with little $(30 \%)$ subjective improvement. This patient went on to have punctal retrieval surgery with anatomical success but minimal subjective improvement. One patient with Sjögren's syndrome, who had undergone 4-eyelid punctal cautery, had subsequent symptomatic epiphora in one eye, despite Schirmer's with anaesthetic of $2 \mathrm{~mm}$. She requested treatment for this and her epiphora improved after one injection of 2.5 units. She chose not to have repeat treatment as her dry eye symptoms worsened.

\section{Discussion}

Patients with epiphora with canalicular obstruction, those who are elderly or medically frail and those with previous malignancy involving the lacrimal system are particularly challenging. Surgery in the latter group is often a relative contraindication for fear of facilitating tumour spread into the adjacent nose and sinuses. Pharmacologic treatment of this group is a useful adjunct in the evolving armamentarium for symptomatic improvement. It may be cheaper, safer and less invasive than surgery.

This study adds to the scarce evidence in the literature supporting the value of BoNTA in the non-surgical treatment of patients with lacrimal outflow obstruction.

There are only two case series reporting on the application of BoNTA in non-crocodile tear epiphora. Wojno 9 has provided the only existing evidence on the value of BoNTA in 27 patients with lacrimal outflow obstruction. Sixty-three per cent of this group mostly or 


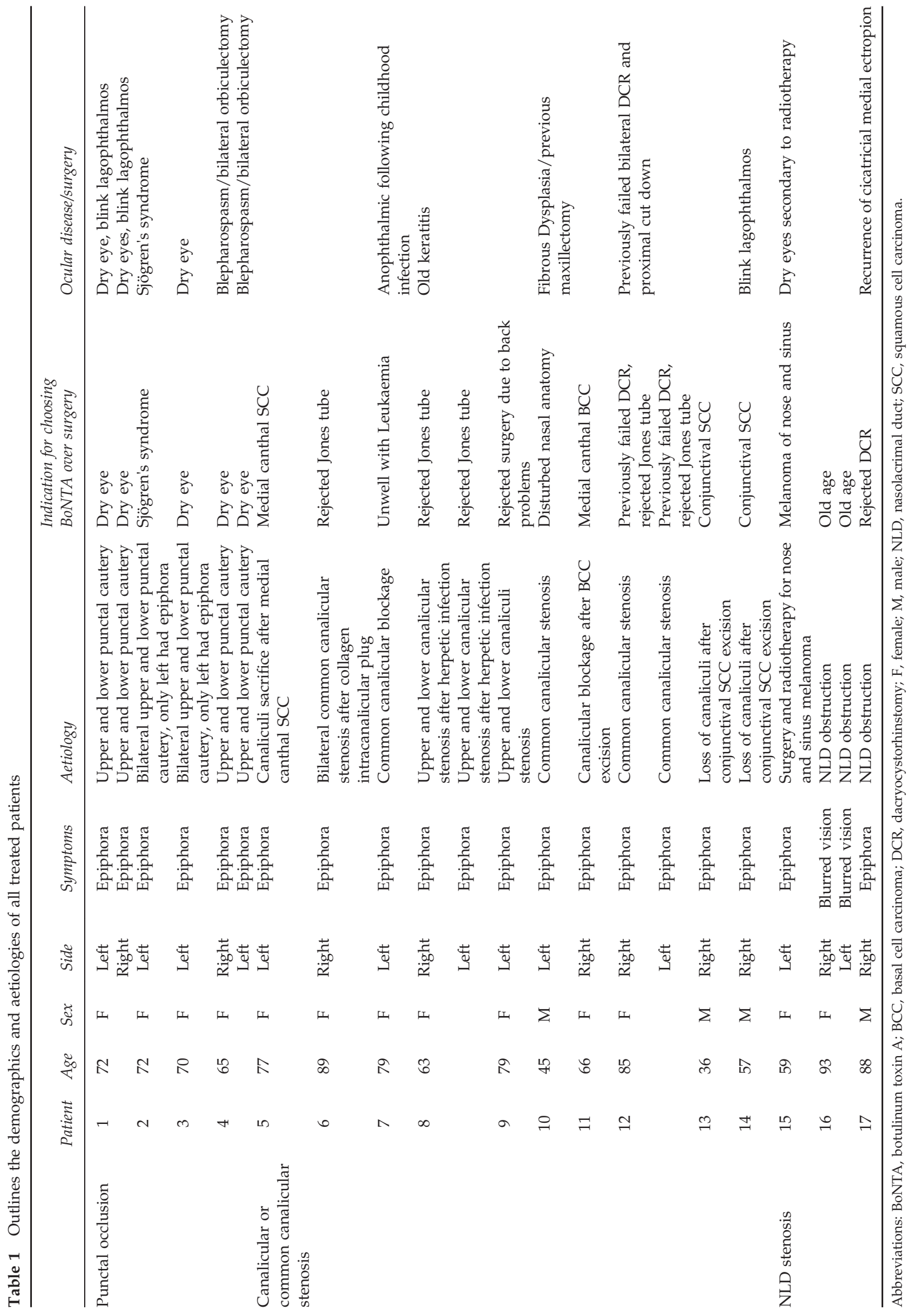


Table 2 Outlines the Munk scores before and after injections

\begin{tabular}{|c|c|c|c|c|c|}
\hline & Patient & $\begin{array}{l}\text { Indoors Munk score } \\
\text { before treatment }\end{array}$ & $\begin{array}{l}\text { Indoors Munk score } \\
\text { after treatment }\end{array}$ & $\begin{array}{c}\text { Outdoors Munk score } \\
\text { before treatment }\end{array}$ & $\begin{array}{c}\text { Outdoors Munk score } \\
\text { after treatment }\end{array}$ \\
\hline \multirow[t]{6}{*}{ Punctal occlusion } & 1 & 4 & 3 & 4 & 3 \\
\hline & - & 4 & 3 & 4 & 3 \\
\hline & 2 & 3 & 0 & 4 & 0 \\
\hline & 3 & 3 & 0 & 4 & 0 \\
\hline & 4 & 4 & 3 & 4 & 3 \\
\hline & - & 4 & 2 & 4 & 2 \\
\hline \multirow{12}{*}{$\begin{array}{l}\text { Canalicular/common } \\
\text { canalicular stenosis }\end{array}$} & 5 & 4 & 1 & 4 & 1 \\
\hline & 6 & 4 & 4 & 4 & 4 \\
\hline & 7 & 3 & $(1,1,1) 1$ & 3 & 2 \\
\hline & 8 & 4 & $(2,1,2,2,1) 1.6$ & 4 & NA \\
\hline & - & 4 & $(2,1,2,2,1) 1.6$ & 4 & NA \\
\hline & 9 & 3 & 1 & 4 & 1 \\
\hline & 10 & 4 & $(3,1,1) 1.6$ & 4 & NA \\
\hline & 11 & NA & NA & NA & \\
\hline & 12 & 3 & 1 & 3 & 1 \\
\hline & - & 2 & 1 & 3 & 1 \\
\hline & 13 & 3 & 1 & NA & NA \\
\hline & 14 & 3 & 1 & & \\
\hline \multirow[t]{4}{*}{ NLD stenosis } & 15 & 4 & 0 & 4 & 1 \\
\hline & 16 & NA & - & - & - \\
\hline & - & NA & - & - & - \\
\hline & 17 & 2 & 3 & 3 & 3 \\
\hline
\end{tabular}

Abbreviations: NA, not available; NLD, nasolacrimal duct.

completely improved with 2.5 units of BoNTA. This improved to $71 \%$ with an additional 2.5 units to those with less than maximal improvement. He did not elaborate on the underlying pathology in these patients. Whittaker $e$ e $a l^{10}$ investigated the usefulness of BoNTA in patients with functional epiphora and achieved symptomatic relief in 8 of 11 patients with transconjunctival injections of 2.5-5 units of BoNTA in the palpebral lobe of the lacrimal gland, causing transient ptosis and diplopia in two patients.

Our results are consistent with the existing evidence with $70 \%$ of patients achieving $>60 \%$ improvement in their symptoms with significant improvement in Munk scores and median duration of effectiveness of 10 weeks. The majority of patients in our series who benefited from this treatment were elderly, a mean 10 years older than those in the series by Wojno. Advanced age, frailty and co-existing morbidities often make attending clinic appointments difficult for this group let alone surgical intervention. Therefore BoNTA is a useful alternative in this group for whom little else may be available.

We found BoNTA beneficial in patients with previous malignancy involving the lacrimal apparatus. Five patients (age range 36-77) had canalicular or NLD obstruction following excision of the carcinoma of their eyelid or conjunctiva and melanoma of the nose and sinus. All reported a significant improvement in their symptoms.
That patients in our series who were initially given doses $>2.5$ units with no complications and subsequently achieved the same improvement with 2.5 unit injections suggests that doses $>2.5$ units may not produce superior outcomes. Although we did not observe any side effects with the higher dose, symptoms in one patient with NLD obstruction and recurrent cicatricial ectropion deteriorated after 2.5 units. This patient improved after ectropion repair, suggesting that eyelid malposition should be addressed first in patients being considered for this option.

We treated two patients with dry eye syndrome and symptomatic epiphora following punctal cautery. One patient experienced a deterioration of dry eye symptoms and the other reported minimal improvement despite punctal retrieval. This emphasises the importance of counselling patients with severe dry eye against interventions to improve epiphora.

BoNTA has been used safely for decades with no longterm side effects. Demetriades $e t a l^{12}$ reported no evidence of histological changes in the lacrimal gland of rabbit following injection of 1.25 and 2.5 units of BoNTA. This is similar to previous reports confirming the absence of orbicularis histological changes following BoNTA injection for blepharospasm. ${ }^{13}$

In conclusion, our results show favourable outcomes for non-surgical BoNTA treatment of lacrimal outflow obstruction in the elderly patients or those for whom 


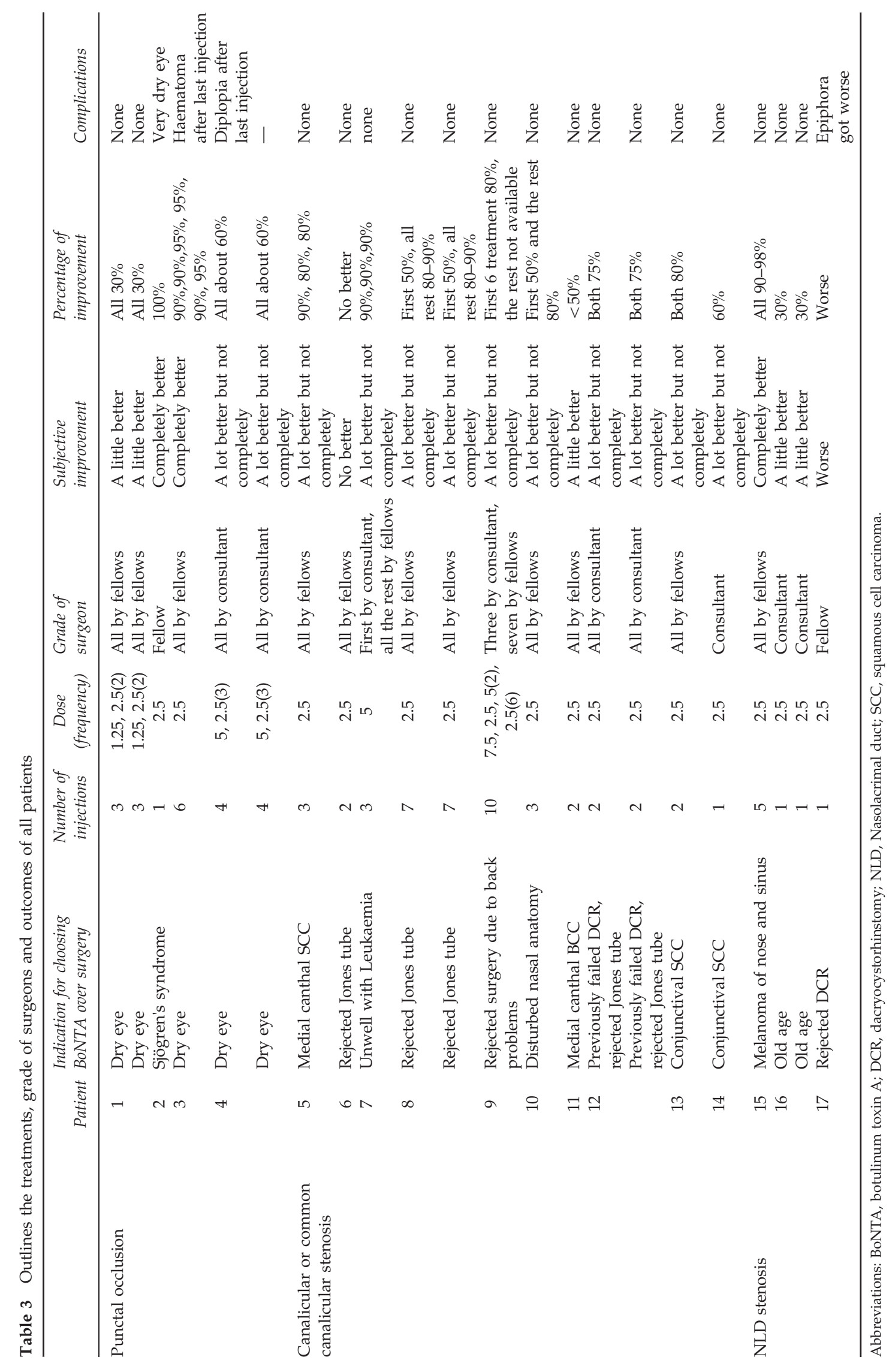


surgery may be contraindicated. Epiphora completely resolved in a fifth, improved by up to $60-90 \%$ in a half and only 'a little better' in a further fifth. This information may be of value for informed consent and for commissioning organisations considering funding this therapeutic option.

\section{Summary}

\section{What was known before}

- Surgery for lacrimal outflow obstruction may not be the best option in all patients especially in the elderly and medically frail patients and after excision of lacrimal apparatus for treatment of malignant lesions affecting the neighbouring anatomical structures.

- Botulinum toxin has been successfully used to treat gustatory hyperlacrimation owing to an aberrant regeneration in proximal facial nerve injuries.

- There is scarce evidence in the literature showing the usefulness of BoNTA in patients with functional epiphora and lacrimal outflow obstruction.

\section{What this study adds}

- We report favourable outcomes for non-surgical BoNTA treatment of lacrimal outflow obstruction.

- Epiphora completely resolved in a fifth, improved by up to $60-90 \%$ in a half and only 'a little better' in a further fifth.

\section{Conflict of interest}

The authors declare no conflict of interest.

\section{References}

1 Rosen N, Ashkenazi I, Rosner M. Patient dissatisfaction after functionally successful conjunctivodacryocystorhinostomy with Jones tube. Am J Ophthalmol 1994; 117: 636-642.
2 Lim C, Martin P, Benger R, Kourt G, Ghabrial R. Lacrimal canalicular bypass surgery with the Lester Jones tube. Am J Ophthalmol 2004; 137: 101-108.

3 Sekhar GC, Dortzbach RK, Gonnering RS, Lemke BN. Problems associated with conjunctivodacryocystorhinostomy. Am J Ophthalmol 1991; 112: 502-506.

4 Stern ME, Beuerman RW, Fox RI, Gao J, Mircheff AK, Pflugfelder SC. The pathology of dry eye: the interaction between the ocular surface and lacrimal glands. Cornea 1998; 17: $584-589$.

5 Boroojerdi B, Ferbert A, Schwarz M, Herath H, Noth J. Botulinum toxin treatment of synkinesia and hyperlacrimation after facial palsy. J Neurol Neurosurg Psychiatry 1998; 65: 111-114.

6 Riemann R, Pfennigsdorf S, Riemann E, Naumann M. Successful treatment of crocodile tears by injection of botulinum toxin into the lacrimal gland: a case report. Ophthalmology 1999; 106: 2322-2324.

7 Hofmann RJ. Treatment of Frey's syndrome (gustatory sweating) and 'crocodile tears' (gustatory epiphora) with purified botulinum toxin. Ophthal Plast Reconstr Surg 2000; 16: 289-291.

8 Falzon K, Galea M, Cunniffe G, Logan P. Transconjunctival botulinum toxin offers an effective, safe and repeatable method to treat gustatory lacrimation. Br J Ophthalmol 2010; 94: 379-380.

9 Wojno TH. Results of lacrimal gland botulinum toxin injection for epiphora in lacrimal obstruction and gustatory tearing. Ophthal Plast Reconstr Surg 2011; 27: 119-121.

10 Whittaker KW, Matthews BN, Fitt AW, Sandramouli S. The use of botulinum toxin A in the treatment of functional epiphora. Orbit 2003; 22: 193-198.

11 Munk PL, Lin DT, Morris DC. Epiphora: treatment by means of dacryocystoplasty with balloon dilation of the nasolacrimal drainage apparatus. Radiology 1990; 177: 687-690.

12 Demetriades AM, Leyngold IM, D'Anna S, Eghrari AO, Emmert DG, Grant MP, Merbs SL. Intraglandular injection of botulinum toxin a reduces tear production in rabbits. Ophthal Plast Reconstr Surg 2013; 29: 21-24.

13 Harris CP, Alderson K, Nebeker J, Holds JB, Anderson RL. Histologic features of human orbicularis oculi treated with botulinum A toxin. Arch Ophthalmol 1991; 109: 393-395. 